\title{
PROPANE SIMULATED IN SILICA PORES: ADSORPTION \\ ISOTHERMS, MOLECULAR STRUCTURE, AND MOBILITY
}

\author{
Thu Le and Alberto Striolo* \\ Department of Chemical Engineering, University College London, London WC1E 7JE, United Kingdom \\ David R. Cole \\ School of Earth Sciences, Ohio State University, Columbus, Ohio 43210, United States
}

\begin{abstract}
Molecular dynamics simulations were conducted for bulk propane in contact with fully protonated slitshaped silica pores. The effective pore width was of either 0.8 or $2.7 \mathrm{~nm}$. The temperature was set at 343 , 368, and 373K. The TraPPE-UA and CLAYFF force fields were implemented to model propane and silica, respectively. Each individual simulation yields the density of confined fluid as a function of the bulk pressure. For a given temperature, adsorption isotherms were estimated by repeating the simulations at various bulk pressures. The results qualitatively agree with available experimental data; namely, at fixed temperature the excess sorption is found to show a maximum near the pressure at which the pores fill; at fixed pressure the excess adsorption is found to decrease as the temperature increases and as the pore width expands. At equilibrium, pronounced layering was observed for propane near the pore surface, especially in the narrower pore and at the highest densities considered. The propane molecules at contact with silica tend to lay with their $\mathrm{CH}_{3}-\mathrm{CH}_{3}$ vector parallel to the pore surface. The mean square displacement as a function of time was used to quantify the self-diffusion coefficient of confined propane as a function of temperature, pressure and pore width. These results will be useful for enhancing the interpretation of experimental data.
\end{abstract}

* Author to whom correspondence should be addressed: a.striolo@ucl.ac.uk

Keywords: Molecular dynamics simulations; Adsorption; Structure; Diffusion 


\section{INTRODUCTION}

Nanoporous materials offer unique properties due to their high specific surface area and large specific pore volume. These properties render them suitable for applications in catalysis, gas storage, separation and purification, among others. Although methane is the most abundant component of natural gas, higher molecular weight hydrocarbons, such as ethane and propane, are much more economically attractive in the current economic landscape (Jackson et al., 2013). This work focuses on propane. Understanding the molecular features related to propane adsorption and diffusion in narrow silica-based pores might be useful for quantifying the molecular phenomena that occur in industrial processes such as catalytic conversions, and also during natural gas production following hydraulic fracturing. Several sub-surface phenomena, including hydrocarbon migration, could be better understood and predicted once the adsorption and diffusion of hydrocarbons in narrow pores are elucidated (Yethiraj and Striolo, 2013).

The focus here is on adsorption isotherms for propane in narrow slit-shaped silica pores, for which experimental data are available. Grande and Rodrigues (Grande and Rodrigues, 2001) measured propane adsorption in silica gel (NSG and WSG, with average pore diameter of 4.4 and $12 \mathrm{~nm}$, respectively). Up to $100 \mathrm{kPa}$, their results are consistent with type I isotherms (Rouquerol et al., 1994; Sing et al., 1985). In 2012, Gruszkiewicz et al. (Gruszkiewicz et al., 2012) used the vibrating tube densimeter to measure propane adsorption isotherms in hydrophobic silica aerogels with average pore size between 7 and $9 \mathrm{~nm}$ (although it is possible that the materials used had pores of size as large as $15 \mathrm{~nm}$ ). Their results were interpreted as a function of the excess adsorption. Rother et al. (Rother et al., 2007) used small angle neutron scattering, SANS, to study experimentally the adsorption of deuterated propane at super-critical temperatures in silica aerogels with $96 \%$ porosity and specific surface area of $\sim 700 \mathrm{~m}^{2} / \mathrm{g}$.

Often complementary to experiment, classical density functional theory (DFT) and molecular simulations are employed to predict the adsorption isotherms of fluids in model porous materials. Monson recently provided an overview on how classical DFT can be used to predict fluid adsorption/desorption hysteresis in mesopores (Monson, 2012). This technique has been proven to be an efficient and accurate method in 
investigating the structure and thermodynamics of confined fluids, the density distribution during adsorption/desorption process, and also in extracting the pore size distribution from experimental adsorption isotherms (Ravikovitch et al., 1998; Wu and Li, 2007). In some cases, molecular simulations can enhance theoretical predictions (Duren et al., 2009; Sarkisov and Monson, 2001). A method often used to simulate adsorption isotherms is the grand canonical Monte Carlo (GCMC) algorithm (Nicholson and Parsonage, 1982). Examples include water adsorption in carbons (Jorge et al., 2002; Liu and Monson, 2006; McCallum et al., 1998; Striolo et al., 2003; Striolo et al., 2005) and in silica pores (Puibasset and Pellenq, 2005) and simple fluids adsorption in various adsorbents (Abbott and Colina, 2011; Gavalda et al., 2002; Hung et al., 2007; Larsen et al., 2011; Macedonia et al., 2000; Müller et al., 2000; Turner, 1998; Walton et al., 2007; Yazaydın et al., 2009). Fewer studies have employed molecular dynamics (MD) simulations to calculate adsorption isotherms, as this method is better suited to study the transport and structural properties of bulk and confined fluids (Awati et al., 2013; Lee and Rasaiah, 1996; Rajput et al., 2012). Feng et al., (Feng et al., 2013) for example, investigated the properties of some alkanes, including propane, over a wide range of temperature at high pressures in the bulk phase.

In the present work, we use equilibrium MD simulations to study propane adsorption isotherms in slitshaped silica pores. The results are used to qualitatively interpret the experimental adsorption isotherms reported by Gruszkiewicz et al. (Gruszkiewicz et al., 2012) and to support part of the insights obtained using SANS by Rotheret et al. (Rother et al., 2007). We report details concerning structural (i.e., density profiles and molecular orientation) and dynamic properties (i.e., self-diffusion coefficients and residence time at contact with the pore surface) of confined propane. We consider thermodynamic conditions at which bulk propane is sub-, near-, and super-critical. Desorption is not investigated.

In the next section, we introduce the simulation methodology and explain the force fields implemented. We then discuss our results and finally summarize the implications of our study. 


\section{SIMULATION METHODOLOGY}

Molecular dynamics (MD) simulations were carried out to compute adsorption isotherms, density profiles, molecular orientation, mean square displacements, and residence autocorrelation functions for propane in fully protonated silica slit-shaped pores. The simulations were conducted at 343, 368, and 373K, which are below, near, and above the propane bulk critical temperature, respectively. Note that the experimental critical temperature for propane (369.8K) (Lemmon et al., n.d.) differs from that predicted by simulations ( 368K) (Teja et al., 1990) when the TraPPE-UA force field is used.

The silica substrate was modeled to replicate $\beta$-cristobalite. The initial configuration was built from 8640 Si and $17760 \mathrm{O}$ atoms, and was divided into two parallel slabs to prepare the slit-shaped pore. Two pore widths were considered. The corresponding simulation box sizes were of $18.4 \times 9.95 \times 4.86$ and18.4x9.95x6.86 $\mathrm{nm}^{3}$, respectively. The slit pore was located in the middle of the simulation box, along the $\mathrm{Z}$ direction. The non-bridging oxygen atoms present on the two pore surfaces were fully protonated with a total of $960 \mathrm{H}$ atoms. The solid substrate bears no net charge. Note (Fig. 1) that the simulation box size along the $\mathrm{X}$ direction is larger than the solid substrate. This is done to allow the formation of an equilibrated bulk fluid phase.

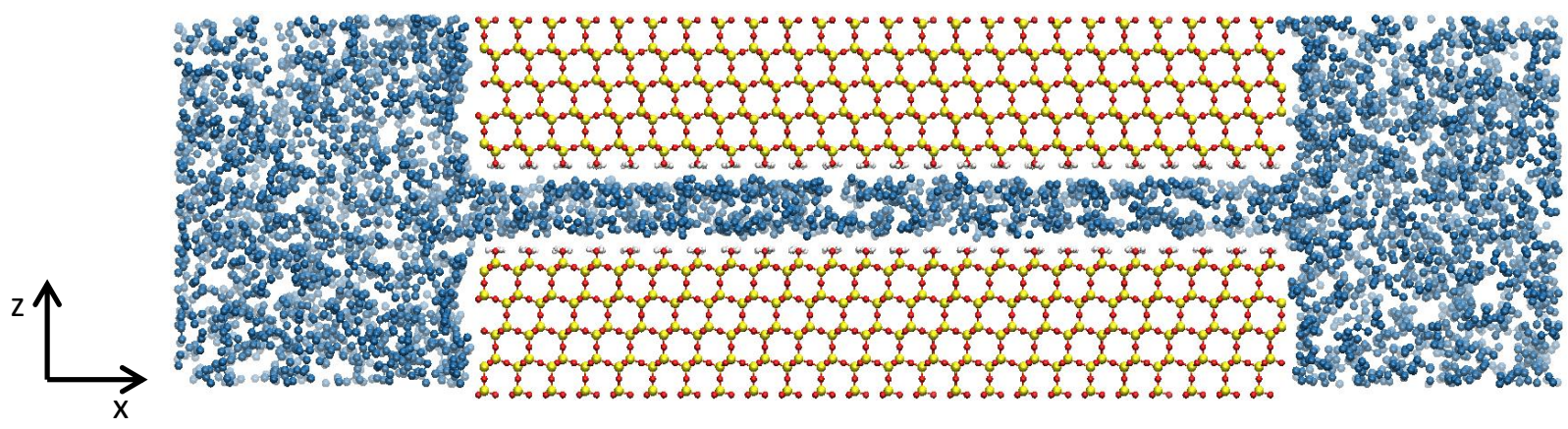

Fig. 1. Schematic representation of the simulation box used for simulating adsorption isotherms. Blue spheres are $\mathrm{CH}_{2}$ and $\mathrm{CH}_{3}$ groups in $\mathrm{C}_{3} \mathrm{H}_{8}$, red is $\mathrm{O}$, white is $\mathrm{H}$, and yellow is $\mathrm{Si}$. 
In Fig. 1, we present the side view of an equilibrated system containing 2000 propane molecules with a slit-shaped silica pore at $\mathrm{T}=368 \mathrm{~K}$. All simulations were carried out within orthorhombic simulation boxes of constant size $(X=18.4 \mathrm{~nm}, \mathrm{Y}=9.95 \mathrm{~nm}$ and $\mathrm{Z}=4.86 \mathrm{~nm}$ for the narrow pore or $\mathrm{Z}=6.86 \mathrm{~nm}$ for the wider pore), containing a constant number of molecules at fixed temperature (i.e., the NVT ensemble). Because periodic boundary conditions (PBCs) were applied in all directions, the systems considered are composed of infinite solid silica slabs along the $\mathrm{Y}$ direction, and separated along the $\mathrm{Z}$ direction by the slit-shaped pore. Along the $\mathrm{X}$ direction the silica substrate is $\sim 10.4 \mathrm{~nm}$ in length, and the pore has two pore entrances. To quantify the adsorption isotherms we only considered the propane molecules that were at least $3 \mathrm{~nm}$ away from the pore entrances. Detailed analysis (not shown for brevity) shows that in the 4-nm wide region near the center of the pores along the $\mathrm{X}$ direction the propane density is constant.

The simulations were initiated with a desired number of propane molecules arranged outside the pore. As the simulations progress, some of the propane spontaneously fill the space around and between the silica slabs. Densities of bulk and confined fluids were calculated at different simulation times. Energy profiles (total energy vs. time) and temperature profiles (temperature vs. time) were also computed (not shown for brevity). Equilibrium was considered achieved when (1) the propane densities reached constant values (+/- 5\%); and (2) both energy and temperature variations remained within $10 \%$ of their respective averaged values. Equilibration was achieved after $12 \mathrm{~ns}$ of simulation time for all systems investigated.

To determine the bulk propane density, we considered the simulation box volume outside of the pore (along the $\mathrm{X}$ direction). The propane density was calculated from silica pore entrance (located at $\mathrm{X}=4 \mathrm{~nm}$ in Fig. 2) into the 'bulk' fluid (towards $X=0 \mathrm{~nm}$ in Fig. 2). For each simulated system, we observed an accumulation of propane near the solid, while the propane density approached an approximately constant value far from the solid. This constant density (from $X=0$ to $X \sim 2.5 \mathrm{~nm}$ in Fig. 2) was considered as the equilibrium bulk density, $\rho_{b}$, for each system. 


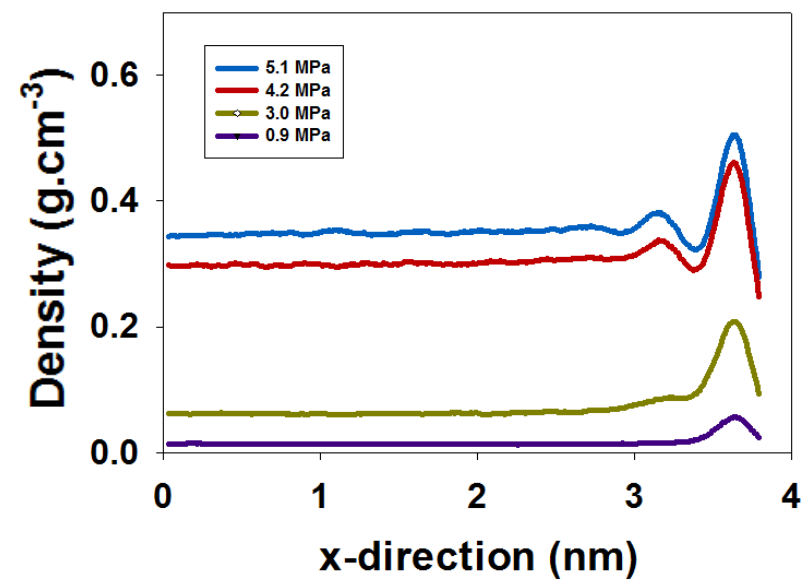

Fig. 2. Density profiles of propane along the $X$ direction of the simulation box, outside of the pore. The solid is located at $\mathrm{X}=4 \mathrm{~nm}$ in this figure. The simulations shown here were conducted for the pore of width $2.7 \mathrm{~nm}$ at $368 \mathrm{~K}$. Different curves represent results at different numbers of propane molecules, which result in different bulk pressures, as summarized in the caption.

Our simulations yield the total amount of adsorbate molecules contained within the pore, $n_{\text {abs }}$. We calculate the excess amount adsorbed, $n_{e x}$, as:

$$
n_{e x}=n_{a b s}-V_{p} \rho_{b}
$$

In Eq. (1), $V_{p}$ is the effective pore volume and $\rho_{b}$ is the propane density in the bulk. To estimate $V_{p}$ we reduce the nominal pore width $H$ (the distance between the centers of the solid atoms across the pore surface, which corresponds to 1 and $3 \mathrm{~nm}$ in the two pores considered here) to obtain the effective pore width, $H^{\prime}$ (Do and Do, 2005; Everett and Powl, 1976; Kaneko et al., 1994):

$$
H^{\prime}=H-2 z_{0}+\sigma_{f f}
$$

In Eq. (2), for $\sigma_{f f}$ we used the averaged Lennard-Jones parameter of ethyl and methyl groups in propane, $\sim 0.385 \mathrm{~nm}$, and for $z_{0}$ we used the distance at which the interaction potential between solid silica and a methyl group equals zero, $\sim 0.292 \mathrm{~nm}$ and $0.323 \mathrm{~nm}$ for the 1 and $3 \mathrm{~nm}$ wide pores, respectively. The 
resultant $H^{\prime}$ values are 0.801 and $2.739 \mathrm{~nm}$ (indicated as effective pore widths of 0.8 and $2.7 \mathrm{~nm}$ in the text). The effective pore volumes within which propane adsorbed were of $\sim 32$ and $109 \mathrm{~nm}^{3}$, respectively.

From the bulk density $\rho_{b}$ at a given temperature (see Fig. 2) we extracted the corresponding bulk pressure by referring to the experimental isothermal properties of fluid systems from the National Institute of Standards and Technology (NIST) database (Lemmon et al., n.d.). For each bulk pressure estimated, the simulations described in Fig. 1 allowed us to determine the density of propane molecules confined within the slit-shaped pore, and therefore we constructed adsorption isotherms.

Quantifying the dynamic properties of confined propane requires a change in the system configuration. The region outside the pores was removed, and because of periodic boundary conditions the slit-shaped pores became effectively infinite along both $\mathrm{X}$ and $\mathrm{Y}$ directions. The dimensions of the simulation boxes are $10.4 \times 9.95 \times 4.86$ and $10.4 \times 9.95 \times 6.86 \mathrm{~nm}^{3}$ for the two pores. A number of propane molecules, corresponding to a desired bulk pressure value, were arranged inside the pore to initiate the simulations. We found that only $\sim 8 \mathrm{~ns}$ were necessary for the systems to reach equilibrium (constant system energy and constant density distribution inside the pore, data not shown).

The CLAYFF (Cygan et al., 2004) force field was implemented to simulate silica. All the atoms of silica were maintained rigid. To simulate propane we refer to Zhao et al. (Zhao et al., 2002), who calculated the thermodynamic properties of pure propane at various thermodynamic conditions using five different force fields. Their results suggest that TraPPE-UA is the most accurate force field for describing propane adsorption isotherms on graphitic pores as well as for calculating liquid and gas phase pressures. Based on these observations, the TraPPE-UA force field (Martin and Siepmann, 1998) was employed here to simulate propane. Dispersive interactions were modeled with the $12-6$ Lennard-Jones potential with parameters for non-like components obtained using Lorentz-Berthelot mixing rules $\left[\varepsilon_{\mathrm{ij}}=\left(\varepsilon_{\mathrm{i}} \varepsilon_{\mathrm{j}}\right)^{1 / 2}\right.$, $\sigma_{\mathrm{ij}}=\left(\sigma_{\mathrm{i}}+\sigma_{\mathrm{j}}\right) / 2$ ] (Allen, 2004; Berthelot, 1898; Lorentz, 1881). All other parameters were taken from 
literature. Following prior simulations in our group (Phan et al., 2012), the cutoff distance for all interaction was set at $0.9 \mathrm{~nm}$ (in the TraPPE model the cutoff is $1.4 \mathrm{~nm}$ ).

Our simulations were performed in the canonical ensemble (NVT) using GROMACS, version 4.5.5 (Hess et al., 2008; Van Der Spoel et al., 2005). Although each simulation was conducted at constant number of molecules $(\mathrm{N})$, several simulations were conducted for the same pore width at increasing number of propane molecules and constant temperature, T. T was controlled by a Nosé-Hoover thermostat with a relaxation time of 100 fs (Hoover, 1985; Nosé, 1984). When necessary, bond lengths were maintained fixed using the LINCS algorithm (Miyamoto and Kollman, 1992). The systems contain from 70 to 5000 propane molecules. Each simulation was run for 15000000 steps with a time step of $0.001 \mathrm{ps}$, for a total of $15 \mathrm{~ns}$. Thermodynamic properties of adsorbed propane were analyzed during the last $3 \mathrm{~ns}$ of the simulations, while dynamical properties were analyzed during the last 1 ns of the corresponding simulations. 


\section{RESULTS AND DISCUSSION}

In Fig. 3 we reproduce, for comparison, the experimental data by Gruszkiewicz et al. (Gruszkiewicz et al., 2012) for the adsorption isotherms for propane in silica aerogels, panel (a), and the corresponding excess adsorption data, panel (b). These experimental results were obtained at 343,368 , and $370 \mathrm{~K}$. These data will be used for qualitative comparison against the simulation results discussed below.
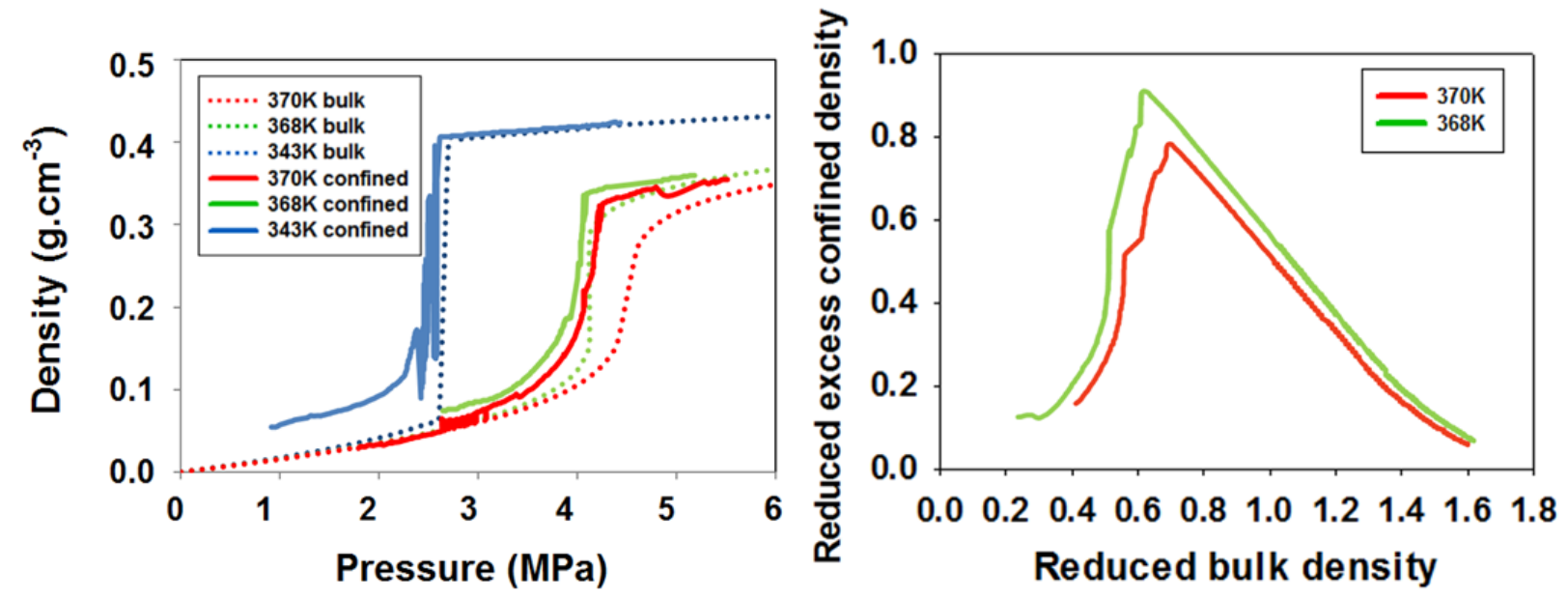

Fig. 3. Experimental adsorption isotherms (a) and reduced excess density of propane confined in silica aerogel (b). The data are adapted from Gruszkiewiczet et al. (Gruszkiewicz et al., 2012).

\subsection{Simulated Adsorption Isotherms}

In Fig. 4, panel (a) we report the simulated adsorption isotherms for propane in the slit-shaped silica pore of width $0.8 \mathrm{~nm}$. The pressure range considered for our simulations is from 0 to $6 \mathrm{MPa}$. Three isotherms were obtained at 343, 368, and 373K. In qualitative agreement with the experiments of Fig. 3, our simulation results show that the confined densities (dots) are always higher than the bulk densities (lines). The excess adsorptions and the excess reduced confined density obtained from simulations are shown in panels (b) and (c), respectively. Reduced densities are estimated as $\rho / \rho_{c}$, where $\rho_{c}=0.22 \mathrm{~g} / \mathrm{cm}^{3}$ for $\mathrm{C}_{3} \mathrm{H}_{8}$. The excess adsorption data show clear maxima. At low pressures, both confined and bulk propane are at 
low density, and the excess adsorption is low. At high pressures, both confined and bulk propane densities are high, and the excess adsorption is low. Although sometimes both experimental (Gruszkiewicz et al., 2012; Rother et al., 2007) and simulated (Do et al., 2010; Myers and Monson, 2002) results can show negative excess adsorption at high pressures, this is not the case for our simulations. Do et al. (Do et al., 2010) suggested that an overestimation as small as $2 \%$ of accessible volume can result in negative excess sorption. At intermediate pressures, the density of both confined and bulk propane increases. In the bulk this increase is a consequence of the gas-to-liquid transition, when $\mathrm{T}$ is below the critical temperature. In the pore this increase is a consequence of pore filling (either primary, i.e., continuous, or due to capillary condensation, depending on the pore width, (Thommes, 2010)). When, as in the systems considered here, pore filling occurs at pressures lower than those at which the gas-to-liquid transition occurs in the bulk, the excess adsorption shows a maximum. Because at super-critical conditions the transition from low density to high density bulk propane is gradual, the maximum in excess adsorption is narrower and steeper as the temperature is farther below the bulk critical temperature, and it becomes gradual as $\mathrm{T}$ increases. 

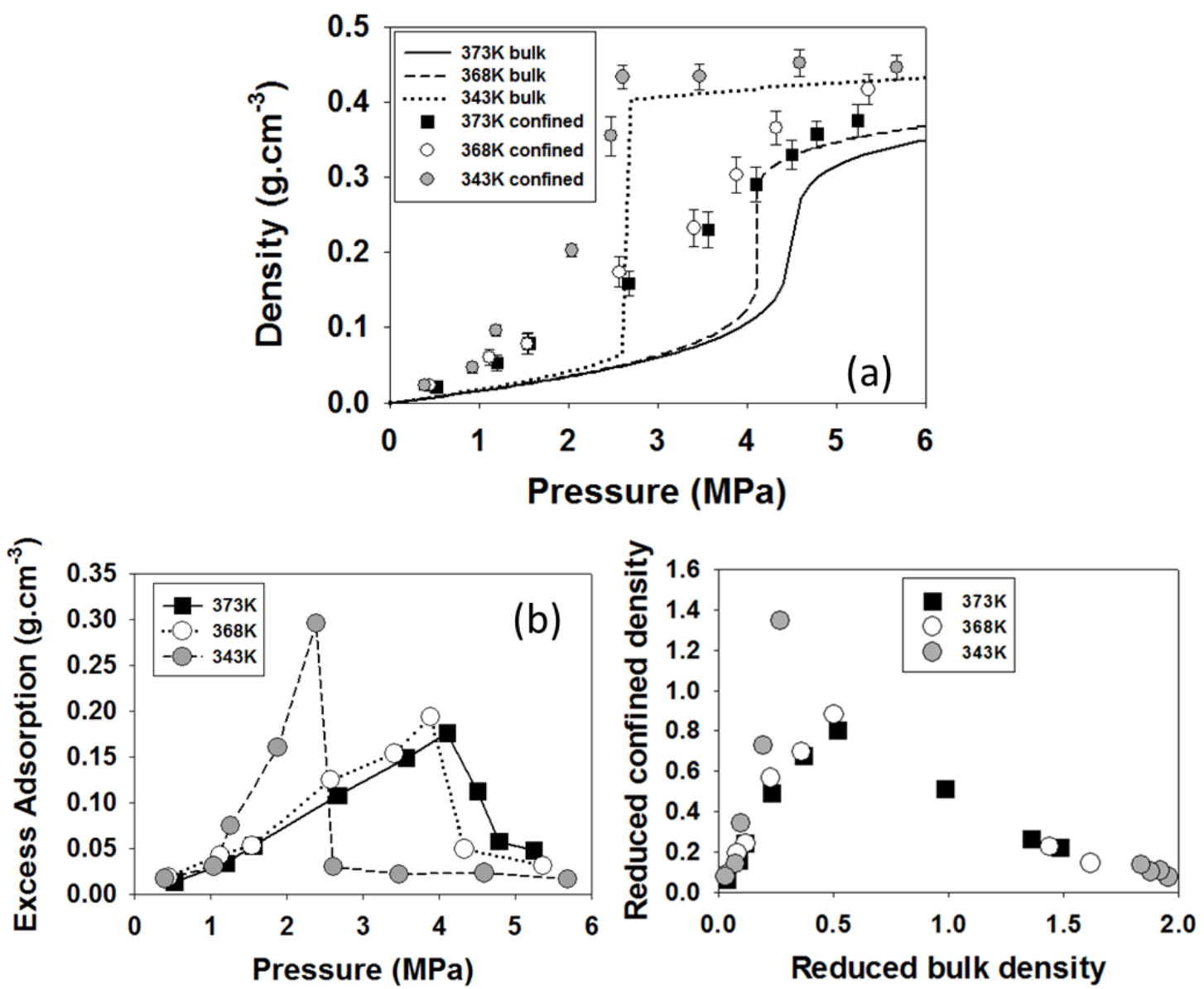

Fig. 4. (a) Simulated densities of propane confined in the $0.8 \mathrm{~nm}$ silica pore (dots) and bulk (lines) propane density; (b) simulated excess adsorption as a function of bulk P; and (c) simulated excess adsorption in reduced terms. Different symbols are for results obtained at 343, 368 and 373K. Isotherms are calculated in the pressure range from 0 to $6 \mathrm{MPa}$. The lines in panel (b) are guides to the eyes.

In Fig. 5, panel (a), the simulated adsorption isotherms for propane at $368 \mathrm{~K}$ for pores of width $0.8 \mathrm{~nm}$ and $2.7 \mathrm{~nm}$ are compared. At low pressures the results exhibit higher density for the propane confined in the narrower pore, as expected. In panel (b), the same data are shown in terms of the excess adsorption. These results show a maximum at intermediate pressures for both pores. As expected based on the discussion above, the maximum is more pronounced for the narrower pore, and the maximum in the wider pore is only slightly shifted to pressures lower than the gas-liquid transition in the bulk. 

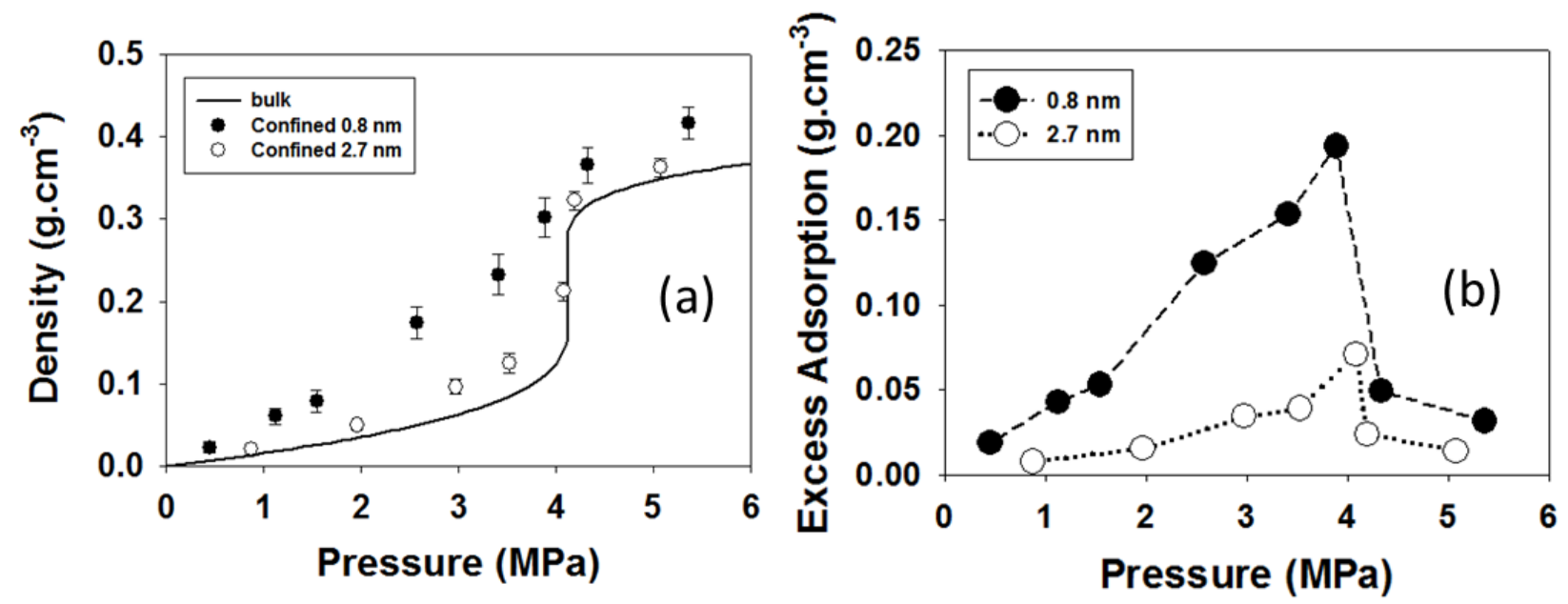

Fig. 5. (a) Simulated adsorption isotherms of confined propane and (b) the corresponding excess adsorption in $0.8 \mathrm{~nm}$ and $2.7 \mathrm{~nm}$ silica pores at $368 \mathrm{~K}$. For comparison, in panel (a) we report the density for bulk propane at $368 \mathrm{~K}$ (line). The lines in panel (b) are guides to the eye.

Although our simulation results for both adsorption isotherms and excess sorption are in qualitative agreement with experimental data (Gruszkiewicz et al., 2012; Rother et al., 2007), some differences are observed. Quantitative agreement between simulations and experiments cannot be expected, as in our simulations the pores are of width 0.8 and $2.7 \mathrm{~nm}$, while the experimental materials have pores of size in the range 7-9 nm, or perhaps even larger. Based on the results in Fig. 5, the difference in pore sizes leads us to expect that the excess adsorption maxima should be more pronounced in our simulated than in the experimental data. However, this is not the case. In fact, our results for the $0.8 \mathrm{~nm}$ pore are comparable to those obtained from experiments. Several physical reasons might be responsible for this discrepancy. The porous materials considered in our work have their non-bridging oxygen atoms fully protonated (hence they are expected to be 'hydrophilic') (Argyris et al., 2009), while the experimental materials were described as 'hydrophobic'. However, because propane does not bear any partial charges, this difference should not lead to important consequences. The geometry of the pores differs in the two sets of data, as the pores are slit-shaped in our simulations, and highly fractal cylinders in the experimental material. The 
pore size distribution, typical of experimental adsorbents, will affect the data, and the pore geometry will have a strong effect on the structural and dynamical properties of the confined fluids. Because, as shown below, our simulations suggest that the excess adsorption is predominantly a surface effect, it is possible that the nature of the surfaces used in simulations and experiments is responsible for some of the differences between simulated and experimental observations. The experiments were conducted in materials with broad range of pore sizes. Thus, it is possible that the large amount of propane within the wider pores did not allow the complete characterization of those propane molecules confined within the narrower pores present in the sample, which would be more comparable to the ones simulated here.

\subsection{Molecular Structure of Confined Propane}

The simulation snapshot in Fig. 1 suggests that propane molecules confined in the silica pores form layers near the solid surfaces. To quantify such possibility, we computed atomic density profiles along the direction perpendicular to the pore surface, following established procedures (Ho et al., 2011). Representative results obtained for the 0.8 and $2.7 \mathrm{~nm}$ pores are shown in Fig. 6, where we distinguish between the density of $\mathrm{CH}_{3}$ (panels (a) and (c)) and $\mathrm{CH}_{2}$ (panels (b) and (d)) groups of propane. 

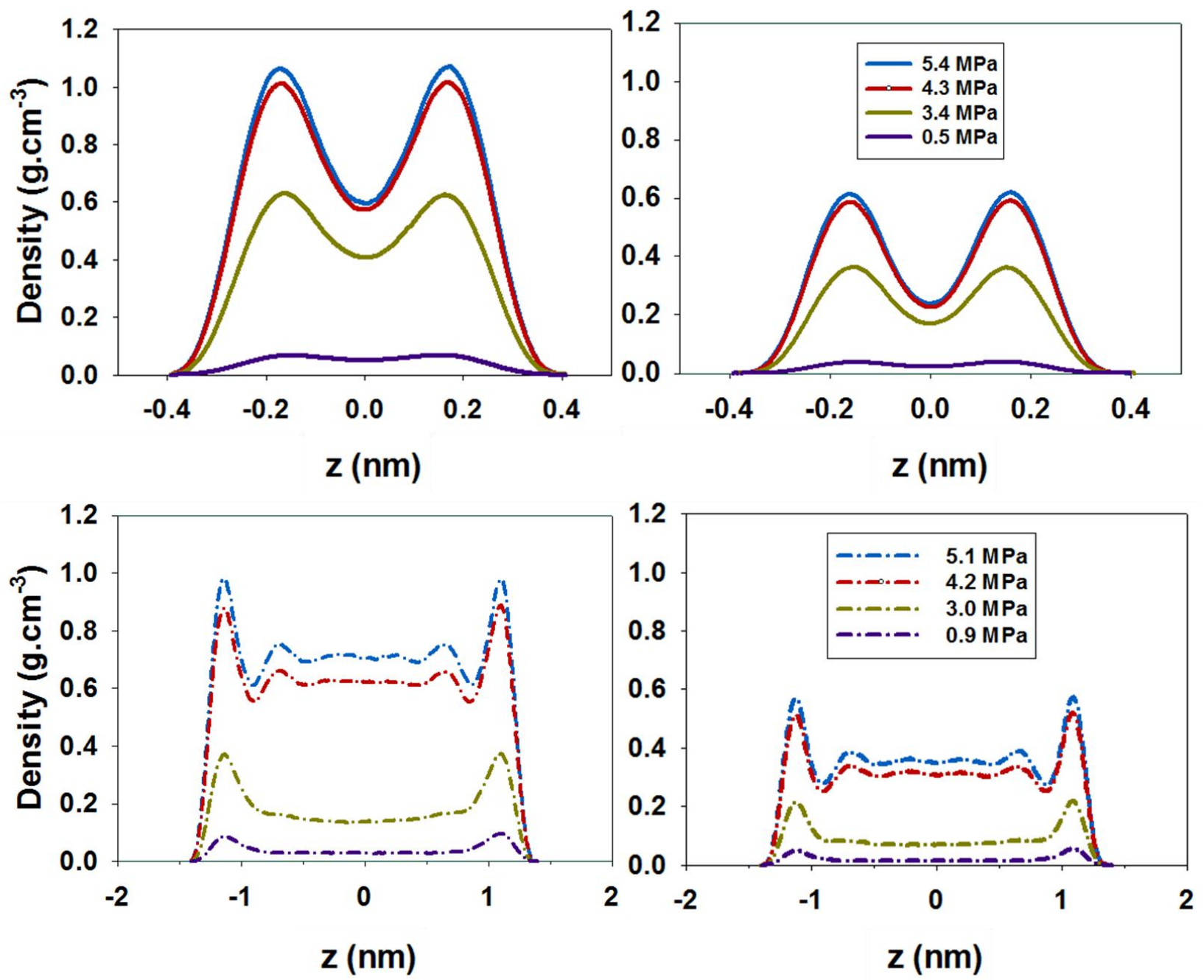

Fig. 6. Atomic density profiles of methyl (left) and ethyl (right) pseudo-atoms of propane confined in 0.8 (top) and 2.7 (bottom) nm silica pores at $368 \mathrm{~K} . \mathrm{Z}$ is the direction perpendicular to the pore surface and $\mathrm{Z}=0$ corresponds to the pore center. Different lines represent results at different bulk pressures.

The results in Fig. 6 were obtained at 368K at increasing bulk pressure. For each curve in panel (a) and (b), where propane is confined in $0.8 \mathrm{~nm}$ pore, there are two distinct peaks with equal heights close to the two silica surfaces (symmetric with respect to the pore center). The peaks are less pronounced as the pressure decreases, as expected. The intensities of the methyl peaks are double those of the ethyl peaks in 
all cases, as the molar ratio for methyl to ethyl is $2: 1$. The fact that methyl and ethyl groups are centered at the same positions suggests that propane molecules lay parallel to the surfaces.

Panel (c) and (d) of Fig. 6 show the atomic density profiles obtained for propane confined within the 2.7 $\mathrm{nm}$ wide silica pores at $368 \mathrm{~K}$. Layering is still observed, although to a much lesser extent than that observed in the $0.8 \mathrm{~nm}$ pore. At high pressures (4.2 and 5.1 MPa), multiple density peaks are visible, suggesting multilayer adsorption, with a rather constant propane density near the pore center. The propane density in this region corresponds, approximately, to the bulk densities at any given pressure. The fact that a part of the pore volume is occupied by propane with density similar to that of bulk propane explains the lower excess adsorption amount observed for this pore, compared to results obtained for the $0.8 \mathrm{~nm}$ pore at similar thermodynamic conditions (see Fig. 5). The density profiles shown in Fig. 6 are in qualitative agreement with the interpretation of SANS data, provided by Rother et al. (Rother et al., 2007) who suggested that an adsorbed phase of thickness 1-2 molecules exists near the silica aerogels.

The density profiles suggest that propane molecules in the first adsorbed layers have the tendency of laying with their $\mathrm{CH}_{3}-\mathrm{CH}_{3}$ vector parallel to the surface. We can quantify this by analyzing the simulation trajectories. We define the propane molecules within the first adsorbed layer as those found within a layer $4 \square$ thick coincident with the density peaks near the surfaces, as identified by Fig. 6. We calculated the angles formed between the $\mathrm{CH}_{3}-\mathrm{CH}_{3}$ vector of the adsorbed molecules and the vector normal to the pore surface. When the angle is $0^{\circ}$ or $180^{\circ}$, the $\mathrm{CH}_{3}-\mathrm{CH}_{3}$ vector is perpendicular to the surface, when it is $90^{\circ}$, the propane lays parallel to the surface. The probability density function for the orientation angle is shown in Fig. 7 for propane in the $0.8 \mathrm{~nm}$ pore at $368 \mathrm{~K}$ and $3.4 \mathrm{MPa}$, panel (a), and in the $2.7 \mathrm{~nm}$ pore at $368 \mathrm{~K}$ and 3.5 MPa, panel (b). In both cases, the results show that propane molecules within the first adsorbed layers on both pores are never perpendicular to the pore surface, and they have a strong preference for laying parallel to the pore surface. In panel (c) of Fig. 7, we provide a representative simulation snapshot for a few propane molecules near the silica surface for visualization purposes. 

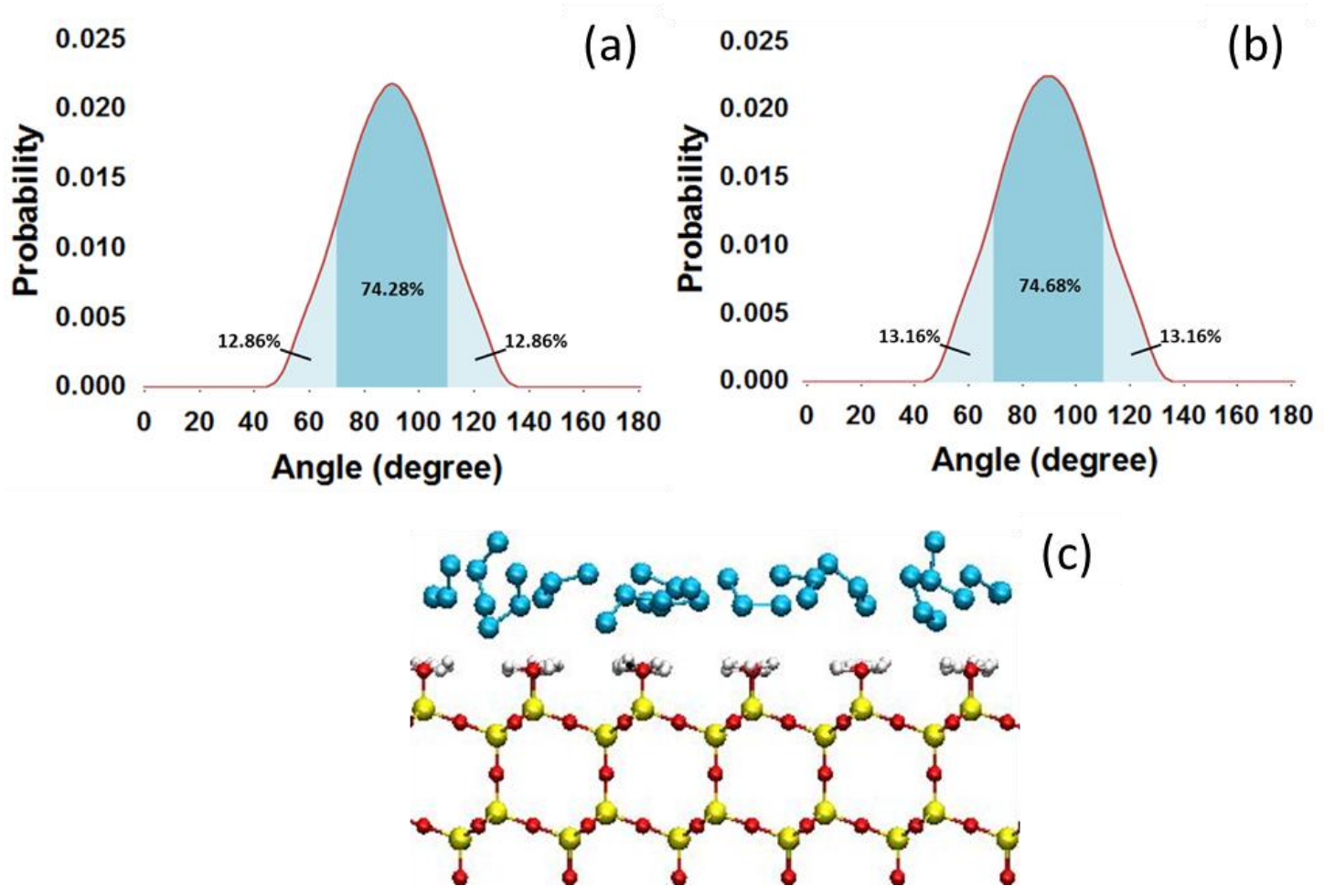

Fig. 7. Probability density function for the orientation of propane molecules adsorbed within the first layer in (a) $0.8 \mathrm{~nm}$ silica pore at $368 \mathrm{~K}$ and $3.4 \mathrm{MPa}$; and in (b) $2.7 \mathrm{~nm}$ pore at $368 \mathrm{~K}$ and $3.5 \mathrm{MPa}$. In panel (c) we provide a representative simulation snapshot for a few propane molecules at contact with the silica surface. The color scheme is the same as that of Fig. 1.

\subsection{Dynamical Properties for Confined Propane}

As the $\mathrm{Z}$ component of the diffusion of propane in the slit-shaped silica pores considered here is negligible, we obtained the planar self-diffusion coefficient $D$ by calculating $1 / 4$ the slope of the propane mean square displacement (MSD) along the $\mathrm{X}$ and $\mathrm{Y}$ directions at long observation times. Calculated propane self-diffusion coefficients are presented in Table 1. Note that for these simulations fixed amounts of propane molecules were simulated in a slit-shaped pore periodic along $\mathrm{X}$ and $\mathrm{Y}$ dimensions. The amount of propane introduced reproduced the expected amount adsorbed at pressures 1, 3, and $5 \mathrm{MPa}$ (see Fig. 4 panel (a) and Fig. 5 panel (a)). 
Table 1. In-plane self-diffusion coefficients estimated for propane confined in silica pore at various temperatures, pressures and pore sizes

\begin{tabular}{ccccc}
\hline \multicolumn{4}{c}{ Propane Planar Self-Diffusion Coefficient $\left(\mathbf{1 0}^{-\mathbf{4}} \mathbf{c m}^{\mathbf{2}} / \mathbf{s}\right)$} \\
\hline \multirow{2}{*}{$\begin{array}{c}\text { Pore size } \\
(\mathbf{n m})\end{array}$} & $\begin{array}{c}\text { Temperature } \\
(\mathbf{K})\end{array}$ & \multicolumn{3}{c}{ Pressure (MPa) } \\
\cline { 2 - 5 } & 343 & $7.2 \pm 0.35$ & $\mathbf{3}$ & $\mathbf{5}$ \\
\hline \multirow{2}{*0.8}{} & 368 & $9.1 \pm 0.45$ & $4.1 \pm 0.05$ & $1.50 \pm 0.05$ \\
\cline { 2 - 5 } & 373 & $10.0 \pm 0.4$ & $4.5 \pm 0.1$ & $2.10 \pm 0.05$ \\
\hline $\mathbf{2 . 8}$ & 368 & $26.9 \pm 1.4$ & $12.1 \pm 0.2$ & $3.3 \pm 0.05$ \\
\hline
\end{tabular}

In the range of investigated thermodynamic conditions, we observe the slowest self-diffusion coefficient for confined propane at the lowest temperature, $343 \mathrm{~K}$, highest pressure, $5 \mathrm{MPa}$, and smaller pore width, $0.8 \mathrm{~nm}$. D increases as temperature and/or pore size increase, and as pressure decreases. These results are expected. In some cases increasing the bulk pressure has little effect on D. For example, at 343K, D obtained in the $0.8 \mathrm{~nm}$ wide pore is similar at 3 and $5 \mathrm{MPa}$. This occurs because the density of confined propane does not change substantially as $\mathrm{P}$ changes in this interval. The results for $\mathrm{D}$ of confined propane are at first sight at odds with experimental data reported by Wang et al. (Wang et al., 2013) for octane and decane diffusivity in microporous BPL activated carbons. At low adsorbate loadings, these authors reported that the adsorbate diffusivity increases with pressure. The interpretation for this observation is as follows. At very low loadings, adsorbate molecules are strongly adsorbed on high-energy adsorption sites, and consequently diffuse slowly. As the amount adsorbed increases, the high-energy adsorption sites are all occupied, and adsorption occurs also on the low-energy adsorption sites. As a consequence, the adsorbate diffusion increases. This behavior is not observed in our system because no high-energy adsorption sites are present for propane on the silica surfaces considered. A recent analysis of the relation between structure and dynamics of simple fluids confined in cylindrical pores has been provided by 
Krekelberg et al. (Krekelberg et al., 2013). The simulation results for this system, in which no preferential adsorption sites exist for the simple fluid, are in qualitative agreement with those presented in Table $\mathbf{1 .}$

For comparison, we calculated the three-dimensional D for propane in bulk systems at temperature and density comparable to the one for the confined fluids. The results are not shown for brevity, but in general the bulk D is larger at low densities, and it becomes comparable, perhaps even slightly lower than D under confinement at high densities. Mittal et al. (Mittal et al., 2006) quantified the relation between selfdiffusion coefficient and density for bulk vs. confined hard spheres. The results were explained in terms of the excess entropy of the fluid molecules, with larger excess entropy leading to larger D. It is possible that, at large density, layering near the surfaces enhances the propane excess entropy, and hence D.

To quantify how long propane molecules remain in contact with the silica surfaces, we computed the residence autocorrelation functions, $\mathrm{C}_{\mathrm{R}}(\mathrm{t})$, for propane molecules within the first adsorption layer within the $2.7 \mathrm{~nm}$ pore. The algorithm is described elsewhere (Phan et al., 2012). We considered the $\mathrm{CH}_{2}$ group of propane to identify the position of one molecule. The more slowly $C_{R}$ decays from 1 to 0 , the longer fluid molecules stay in the adsorbed layer. In Fig. 8, we report the results obtained at various conditions. The results do not show large variations as a function of $\mathrm{T}$ and $\mathrm{P}$. However, the data suggest that propane molecules stay longer at contact with the solid substrate at low T and low P. As T increases at P=3 MPa, as expected (Kleine et al., 1995), $C_{R}$ decreases faster. At $368 K, C_{R}$ decreases faster as P increases. These data suggest that exchanges between propane molecules in the center of the pore and those adsorbed on the surface are facilitated by high propane density in the pore and by high $\mathrm{T}$. 

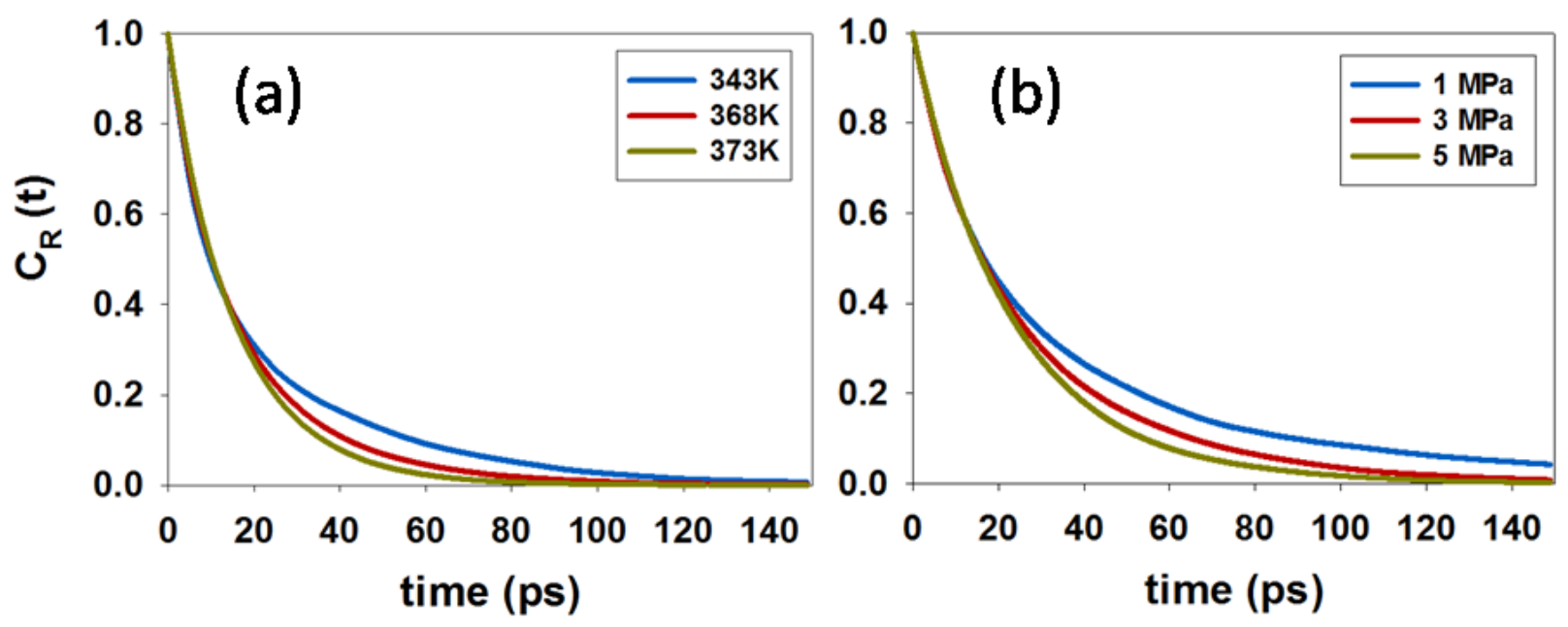

Fig. 8. Residence autocorrelation function $C_{R}(t)$, for propane within the first adsorbed layer within the 2.7 $\mathrm{nm}$ silica pore as a function of (a) temperature at $3 \mathrm{MPa}$, and (b) pressure at $368 \mathrm{~K}$.

\section{CONCLUSIONS}

Molecular dynamics simulations were employed to study structural and dynamic properties of pure propane in slit-shaped fully protonated silica pores at 343,368 , and $373 \mathrm{~K}$. Adsorption isotherms were calculated at varying bulk pressures from 0 to $6 \mathrm{MPa}$ in pores of effective width 0.8 and $2.7 \mathrm{~nm}$. To facilitate comparison with experiments, the results were analyzed in terms of excess adsorption. Despite significant differences between the simulated and experimental systems, reasonable, yet not complete, qualitative agreement was obtained. Some possible reasons have been proposed to explain the discrepancies between simulated and experimental results. We complemented experimental data by investigating the structure of propane molecules adsorbed in the pores and by quantifying some of their transport properties. The highest in-plane self-diffusion coefficients are obtained at high temperature, because of higher molecular mobility, and low pressure, because no high-energy adsorption sites are available for propane within the pores considered in our simulations. The results presented here will be useful to advance applications in the oil and gas, chemical, environmental, and automotive industries. 


\section{ACKNOWLEDGEMENTS}

We appreciate and acknowledge the financial support from the U.S. Department of Energy, Office of Basic Energy Sciences, under Contract Number DE-SC0006901 (Division of Chemical Sciences, Geosciences, and Biosciences). D.R.C. was supported under contract DE-SC0006878 provided by the U.S. Department of Energy, Office of Basic Energy Sciences (Division of Chemical Sciences, Geosciences, and Biosciences). Ms. Thu Le was supported, in part, by the Sloan Foundation, via the Deep Carbon Observatory. Generous allocations of computing time were provided by the Oklahoma Supercomputing Center for Education and Research (OSCER) and by the National Energy Research Scientific Computing Center (NERSC) at Lawrence Berkeley National Laboratory. NERSC is supported by the DOE Office of Science under Contract No. DE-AC02-05CH11231.

\section{REFERENCES}

Abbott, L.J., Colina, C.M., 2011. Atomistic Structure Generation and Gas Adsorption Simulations of Microporous Polymer Networks. Macromolecules 44, 4511-4519.

Allen, M.P.T.D.J., 2004. Computer simulation of liquids. Clarendon Pr., Oxford.

Argyris, D., Cole, D.R., Striolo, A., 2009. Hydration Structure on Crystalline Silica Substrates. Langmuir 25, 8025-8035.

Awati, R.V., Ravikovitch, P.I., Sholl, D.S., 2013. Efficient and Accurate Methods for Characterizing Effects of Framework Flexibility on Molecular Diffusion in Zeolites: $\mathrm{CH} 4$ Diffusion in Eight Member Ring Zeolites. The Journal of Physical Chemistry C 117, 13462-13473.

Berthelot, D., 1898. Sur le mélange des gaz. Comptes rendus hebdomadaires des séances de l'Académie des Sciences, 1703-1855.

Broom, D.P., Thomas, K.M., 2013. Gas adsorption by nanoporous materials: Future applications and experimental challenges. MRS Bulletin 38, 412-421.

Cooke, D.J., Gray, R.J., Sand, K.K., Stipp, S.L.S., Elliott, J.A., 2010. Interaction of Ethanol and Water with the $\{10 \overline{1} 4\}$ Surface of Calcite. Langmuir 26, 14520-14529.

Cygan, R.T., Liang, J.-J., Kalinichev, A.G., 2004. Molecular Models of Hydroxide, Oxyhydroxide, and Clay Phases and the Development of a General Force Field. The Journal of Physical Chemistry B 108, 12551266.

Do, D.D., Do, H.D., 2005. Evaluation of 1-Site and 5-Site Models of Methane on Its Adsorption on Graphite and in Graphitic Slit Pores. The Journal of Physical Chemistry B 109, 19288-19295.

Do, D.D., Do, H.D., Fan, C., Nicholson, D., 2010. On the Existence of Negative Excess Isotherms for Argon Adsorption on Graphite Surfaces and in Graphitic Pores under Supercritical Conditions at Pressures up to 10,000 atm. Langmuir 26, 4796-4806.

Duren, T., Bae, Y.-S., Snurr, R.Q., 2009. Using molecular simulation to characterise metal-organic frameworks for adsorption applications. Chemical Society Reviews 38, 1237-1247. 
Einstein, A., 1905. Über die von der molekularkinetischen Theorie der Wärme geforderte Bewegung von in ruhenden Flüssigkeiten suspendierten Teilchen. Annalen der Physik 322, 549-560.

Everett, D.H., Powl, J.C., 1976. Adsorption in slit-like and cylindrical micropores in the henry's law region. A model for the microporosity of carbons. Journal of the Chemical Society, Faraday Transactions 1: Physical Chemistry in Condensed Phases 72, 619-636.

Feng, H., Gao, W., Nie, J., Wang, J., Chen, X., Chen, L., Liu, X., Lüdemann, H.-D., Sun, Z., 2013. MD simulation of self-diffusion and structure in some $n$-alkanes over a wide temperature range at high pressures. Journal of Molecular Modeling 19, 73-82.

Gavalda, S., Gubbins, K.E., Hanzawa, Y., Kaneko, K., Thomson, K.T., 2002. Nitrogen Adsorption in Carbon Aerogels: A Molecular Simulation Study. Langmuir 18, 2141-2151.

Grande, C.A., Rodrigues, A.E., 2001. Adsorption Equilibria and Kinetics of Propane and Propylene in Silica Gel. Industrial \& Engineering Chemistry Research 40, 1686-1693.

Gruszkiewicz, M.S., Rother, G., Wesolowski, D.J., Cole, D.R., Wallacher, D., 2012. Direct Measurements of Pore Fluid Density by Vibrating Tube Densimetry. Langmuir 28, 5070-5078.

Hess, B., Kutzner, C., van der Spoel, D., Lindahl, E., 2008. GROMACS 4: Algorithms for Highly Efficient, Load-Balanced, and Scalable Molecular Simulation. Journal of Chemical Theory and Computation 4, 435447.

Ho, T.A., Argyris, D., Cole, D.R., Striolo, A., 2011. Aqueous $\mathrm{NaCl}$ and $\mathrm{CsCl}$ Solutions Confined in Crystalline Slit-Shaped Silica Nanopores of Varying Degree of Protonation. Langmuir 28, 1256-1266.

Hoover, W.G., 1985. Canonical dynamics: Equilibrium phase-space distributions. Physical Review A 31, 1695-1697.

Hung, F., Bhattacharya, S., Coasne, B., Thommes, M., Gubbins, K., 2007. Argon and krypton adsorption on templated mesoporous silicas: molecular simulation and experiment. Adsorption 13, 425-437.

Jackson, R.B., Vengosh, A., Darrah, T.H., Warner, N.R., Down, A., Poreda, R.J., Osborn, S.G., Zhao, K., Karr, J.D., 2013. Increased stray gas abundance in a subset of drinking water wells near Marcellus shale gas extraction. Proc. Natl. Acad. Sci. U.S.A.

Jorge, M., Schumacher, C., Seaton, N.A., 2002. Simulation Study of the Effect of the Chemical Heterogeneity of Activated Carbon on Water Adsorption. Langmuir 18, 9296-9306.

Kaneko, K., Cracknell, R.F., Nicholson, D., 1994. Nitrogen Adsorption in Slit Pores at Ambient Temperatures: Comparison of Simulation and Experiment. Langmuir 10, 4606-4609.

Kleine, H., Eckhardt, M., Fick, D., 1995. Mean residence time of Li atoms adsorbed on Si(100) and Si(111) surfaces. Surface Science 329, 71-76.

Krekelberg, W.P., Siderius, D.W., Shen, V.K., Truskett, T.M., Errington, J.R., 2013. Connection between Thermodynamics and Dynamics of Simple Fluids in Highly Attractive Pores. Langmuir 29, 14527-14535. Larsen, G., Lin, P., Siperstein, F., Colina, C., 2011. Methane adsorption in PIM-1. Adsorption 17, 21-26. Lee, S.H., Rasaiah, J.C., 1996. Molecular Dynamics Simulation of Ion Mobility. 2. Alkali Metal and Halide lons Using the SPC/E Model for Water at $25^{\circ} \mathrm{C}+$. The Journal of Physical Chemistry 100, 1420-1425.

Lemmon, E.W., McLinden, M.O., Friend, D.G., n.d. Thermophysical Properties of Fluid Systems. NIST Standard Reference Database 69: NIST Chemistry WebBook. http: //webbook.nist.gov/chemistry/fluid/ (accessed Oct, 2013).

Liu, J.C., Monson, P.A., 2006. Monte Carlo Simulation Study of Water Adsorption in Activated Carbon. Industrial \& Engineering Chemistry Research 45, 5649-5656.

Lorentz, H.A., 1881. Ueber die Anwendung des Satzes vom Virial in der kinetischen Theorie der Gase. Annalen der Physik 248, 127-136. 
Macedonia, M.D., Moore, D.D., Maginn, E.J., Olken, M.M., 2000. Adsorption Studies of Methane, Ethane, and Argon in the Zeolite Mordenite: Molecular Simulations and Experiments. Langmuir 16, 3823-3834.

Martin, M.G., Siepmann, J.I., 1998. Transferable Potentials for Phase Equilibria. 1. United-Atom Description of n-Alkanes. The Journal of Physical Chemistry B 102, 2569-2577.

McCallum, C.L., Bandosz, T.J., McGrother, S.C., Müller, E.A., Gubbins, K.E., 1998. A Molecular Model for Adsorption of Water on Activated Carbon: Comparison of Simulation and Experiment. Langmuir 15, 533-544.

Mittal, J., Errington, J.R., Truskett, T.M., 2006. Thermodynamics Predicts How Confinement Modifies the Dynamics of the Equilibrium Hard-Sphere Fluid. Physical Review Letters 96, 177804.

Miyamoto, S., Kollman, P.A., 1992. Settle: An analytical version of the SHAKE and RATTLE algorithm for rigid water models. Journal of Computational Chemistry 13, 952-962.

Monson, P.A., 2012. Understanding adsorption/desorption hysteresis for fluids in mesoporous materials using simple molecular models and classical density functional theory. Microporous and Mesoporous Materials 160, 47-66.

Morishige, K., Shikimi, M., 1998. Adsorption hysteresis and pore critical temperature in a single cylindrical pore. The Journal of Chemical Physics 108, 7821-7824.

Müller, E.A., Hung, F.R., Gubbins, K.E., 2000. Adsorption of Water Vapor-Methane Mixtures on Activated Carbons. Langmuir 16, 5418-5424.

Myers, A.L., Belfort, G., 1984. Fundamentals of adsorption. Engineering Foundation, NY.

Myers, A.L., Monson, P.A., 2002. Adsorption in Porous Materials at High Pressure: Theory and Experiment. Langmuir 18, 10261-10273.

Nicholson, D., Parsonage, N.G., 1982. Computer simulation and the statistical mechanics of adsorption. Academic Press.

Nosé, S., 1984. A molecular dynamics method for simulations in the canonical ensemble. Molecular Physics 52, 255-268.

Phan, A., Ho, T.A., Cole, D.R., Striolo, A., 2012. Molecular Structure and Dynamics in Thin Water Films at Metal Oxide Surfaces: Magnesium, Aluminum, and Silicon Oxide Surfaces. The Journal of Physical Chemistry C 116, 15962-15973.

Puibasset, J., Pellenq, R., 2005. Water adsorption in disordered mesoporous silica (Vycor) at $300 \mathrm{~K}$ and 650 K: A Grand Canonical Monte Carlo simulation study of hysteresis. The Journal of Chemical Physics 122, 094704.

Rajput, N.N., Monk, J., Hung, F.R., 2012. Structure and Dynamics of an Ionic Liquid Confined Inside a Charged Slit Graphitic Nanopore. The Journal of Physical Chemistry C 116, 14504-14513.

Ravikovitch, P.I., Haller, G.L., Neimark, A.V., 1998. Density functional theory model for calculating pore size distributions: pore structure of nanoporous catalysts. Advances in Colloid and Interface Science 7677, 203-226.

Rother, G., Melnichenko, Y.B., Cole, D.R., Frielinghaus, H., Wignall, G.D., 2007. Microstructural Characterization of Adsorption and Depletion Regimes of Supercritical Fluids in Nanoporest. The Journal of Physical Chemistry C 111, 15736-15742.

Rouquerol, J., Avnir, D., Fairbridge, C.W., Everett, D.H., Haynes, J.M., Pernicone, N., Ramsay, J.D.F., Sing, K.S.W., Unger, K.K., 1994. Recommendations for the characterization of porous solids (Technical Report, IUPAC Recommendations). Pure and Applied Chemistry 66, 1739.

Sarkisov, L., Monson, P.A., 2001. Modeling of Adsorption and Desorption in Pores of Simple Geometry Using Molecular Dynamics. Langmuir 17, 7600-7604.

Sing, K.S.W., Everett, D.H., Haul, R.A.W., Moscou, L., Pierotti, R.A., Rouquerol, J., Siemieniewska, T., 1985. Reporting physisorption data for gas/solid systems with special reference to the determination of surface area and porosity (IUPAC Recommendations 1984). Pure and Applied Chemistry 57, 603-619. 
Striolo, A., Chialvo, A.A., Cummings, P.T., Gubbins, K.E., 2003. Water Adsorption in Carbon-Slit Nanopores. Langmuir 19, 8583-8591.

Striolo, A., Gubbins, K.E., Gruszkiewicz, M.S., Cole, D.R., Simonson, J.M., Chialvo, A.A., Cummings, P.T., Burchell, T.D., More, K.L., 2005. Effect of Temperature on the Adsorption of Water in Porous Carbons. Langmuir 21, 9457-9467.

Teja, A.S., Lee, R.J., Rosenthal, D., Anselme, M., 1990. Correlation of the critical properties of alkanes and alkanols. Fluid Phase Equilibria 56, 153-169.

Thommes, M., 2010. Physical Adsorption Characterization of Nanoporous Materials. Chemie Ingenieur Technik 82, 1059-1073.

Turner, A.R., 1998. A Grand Canonical Monte Carlo Study of Adsorption on Graphite Surfaces with Defects. University of Wales Bangor (Chemistry), UK.

Van Der Spoel, D., Lindahl, E., Hess, B., Groenhof, G., Mark, A.E., Berendsen, H.J.C., 2005. GROMACS: Fast, flexible, and free. Journal of Computational Chemistry 26, 1701-1718.

Walton, K.S., Millward, A.R., Dubbeldam, D., Frost, H., Low, J.J., Yaghi, O.M., Snurr, R.Q., 2007. Understanding Inflections and Steps in Carbon Dioxide Adsorption Isotherms in Metal-Organic Frameworks. Journal of the American Chemical Society 130, 406-407.

Wang, Y., Mahle, J.J., Furtado, A.M.B., Glover, T.G., Buchanan, J.H., Peterson, G.W., LeVan, M.D., 2013. Mass Transfer and Adsorption Equilibrium for Low Volatility Alkanes in BPL Activated Carbon. Langmuir 29, 2935-2945.

Wu, J., Li, Z., 2007. Density-Functional Theory for Complex Fluids. Annual Review of Physical Chemistry $58,85-112$.

Yazaydın, A.O.z.r., Benin, A.I., Faheem, S.A., Jakubczak, P., Low, J.J., Willis, R.R., Snurr, R.Q., 2009. Enhanced $\mathrm{CO} 2$ Adsorption in Metal-Organic Frameworks via Occupation of Open-Metal Sites by Coordinated Water Molecules. Chemistry of Materials 21, 1425-1430.

Yethiraj, A., Striolo, A., 2013. Fracking: What Can Physical Chemistry Offer? The Journal of Physical Chemistry Letters 4, 687-690.

Zhao, X.C., Kwon, S., Vidic, R.D., Borguet, E., Johnson, J.K., 2002. Layering and orientational ordering of propane on graphite: An experimental and simulation study. Journal of Chemical Physics 117, 77197731. 